\title{
New data on the anatomy of fuxianhuiid arthropod Guangweicaris spinatus from the lower Cambrian Guanshan Biota, Yunnan, China
}

Hong Chen, David A. Legg, Da-You Zhai, Yu Liu, and Xian-Guang Hou

Acta Palaeontologica Polonica 65 (1), 2020: 139-148 doi:https://doi.org/10.4202/app.00508.2018

The fuxianhuiid arthropod Guangweicaris spinatus, from the lower Cambrian (Series 2, Stage 4),

Guanshan Biota

(Wulongqing Member, Canglangpu Formation), located in the vicinity of Kunming (Yunnan Province, southwest

China), is redescribed based on new specimens and a re-examination of previously described material. A more complete

overview of its morphology is given. Newly recognised features include: (i) a medial cephalic bulge; (ii) a tripartite

hypostome; (iii) a pair of specialized post-antennal appendage (SPA); (iv) a putative telson; (v) two pairs of spines on

the posteroventral margin of the terminal abdominal segment. This information is used to provide an emended diagnosis

of the family Fuxianhuiidae, and the genus Guangweicaris.

Key words: Arthropoda, Euarthropoda, Fuxianhuiida, Fuxianhuiidae, Guanshan Biota, Cambrian, Asia.

Hong Chen [chenhong3397258@126.com], Yunnan Key Laboratory for

Palaeobiology, Yunnan University, Kunming 650091, China; MEC

International Laboratory for Palaeobiology and Palaeoenvironment,

Yunnan University, Kunming 650091, China; School of Biological

Sciences and Technology, Liupanshui Normal University, Liupanshui,

Guizhou 553004, China. David A. Legg [david.legg@manchester.ac.uk], School of

Earth and Environmental Sciences, University of Manchester, Manchester M13

9PL, UK. Da-You Zhai [dyzhai@ynu.edu.cn], Yu Liu [yu.liu@ynu.edu.cn], and

Xian-Guang Hou [xghou@ynu.edu.cn], Yunnan Key Laboratory for Palaeobiology, Yunnan University, Kunming 650091, China; MEC International Laboratory for Palaeobiology and Palaeoenvironment, Yunnan University, Kunming 650091, China. 
This is an open-access article distributed under the terms of the Creative Commons

Attribution License (for details please see creativecommons.org), which permits unrestricted use, distribution, and reproduction in any medium, provided the original author and source are credited.

\author{
FoF $F$ Full text $(1,542.8 \mathrm{kB})$ । \\ Forif Supplementary file $(562.3 \mathrm{kB})$
}

